\title{
Fatigue in neuromuscular disorders: focus on Guillain-Barré syndrome and Pompe disease
}

\author{
J. M. de Vries • M. L. C. Hagemans • \\ J. B. J. Bussmann · A. T. van der Ploeg • \\ P. A. van Doorn
}

Received: 30 June 2009/Revised: 12 October 2009/ Accepted: 13 October 2009/Published online: 16 November 2009

(C) The Author(s) 2009. This article is published with open access at Springerlink.com

\begin{abstract}
Fatigue accounts for an important part of the burden experienced by patients with neuromuscular disorders. Substantial high prevalence rates of fatigue are reported in a wide range of neuromuscular disorders, such as Guillain-Barré syndrome and Pompe disease. Fatigue can be subdivided into experienced fatigue and physiological fatigue. Physiological fatigue in turn can be of central or peripheral origin. Peripheral fatigue is an important contributor to fatigue in neuromuscular disorders, but in reaction to neuromuscular disease fatigue of central origin can be an important protective mechanism to restrict further damage. In most cases, severity of fatigue seems to be related with disease severity, possibly with the exception of fatigue occurring in a monophasic disorder like Guillain-Barré syndrome. Treatment of fatigue in neuromuscular disease starts with symptomatic treatment of the underlying disease. When symptoms of fatigue
\end{abstract}

J. M. de Vries ( $\square)$

Department of Neurology, Erasmus MC, University Medical Centre, Room Number EE 22-30, Dr. Molenwaterplein 50-60, Postbus 2040, 3000 CA Rotterdam, The Netherlands e-mail: j.m.devries@erasmusmc.nl

J. M. de Vries - M. L. C. Hagemans - A. T. van der Ploeg Division of Metabolic Diseases and Genetics, Department of Paediatrics, Erasmus MC,

Sophia Children's Hospital, Rotterdam, The Netherlands

J. B. J. Bussmann

Department of Rehabilitation Medicine, Erasmus MC, University Medical Centre, Rotterdam, The Netherlands

P. A. van Doorn

Department of Neurology, Erasmus MC, University Medical

Centre, Room Number BA 450,'s Gravendijkwal 230,

Postbus 2040, 3000 CA Rotterdam, The Netherlands persist, non-pharmacological interventions, such as exercise and cognitive behavioral therapy, can be initiated.

Keywords Fatigue - Neuromuscular disease . Experienced fatigue Physiological fatigue Guillain-Barré syndrome · Pompe disease

\section{Introduction}

Everybody experiences periods of fatigue, but these usually can be mended by taking a rest or enjoying a good night's sleep. In a wide range of diseases, however, fatigue is a wellknown chronic symptom. In patients without demonstrable somatic disease chronic fatigue may occur and may lead to the diagnosis of chronic fatigue syndrome [31]. Severe fatigue as a chronic symptom and its impact on daily living are starting to receive more and more attention as patientreported outcome measures in clinical trials in patients with somatic disease. Several studies have demonstrated that fatigue is a disabling symptom in chronic diseases such as cancer, but also in a variety of neurological disorders and conditions (Table 1) [14]. Severe fatigue can also occur as a side effect of drugs like beta-blockers, proton-pump inhibitors, anxiolytic drugs and antipsychotics [2].

The first studies on fatigue in neurological diseases focused on diseases of the central nervous system (CNS), such as cerebrovascular disease, multiple sclerosis and Parkinson's disease [29, 30, 44, 54, 88, 106]. Nowadays, it is recognized that fatigue is also an important and frequently occurring symptom in disorders of the peripheral nervous system (PNS) with a high impact on physical abilities and perceived health status in these patients [104].

This review focuses on fatigue in neuromuscular disorders, with emphasis on fatigue in Guillain-Barré syndrome 
Table 1 Neurological diseases and conditions associated with fatigue

Neurological diseases and disorders associated with fatigue

Cerebral vasculitis and cerebrovascular diseases

Channelopathies

Charcot-Marie-Tooth disease type 1 (CMT type 1)

Chronic inflammatory demyelinating polyneuropathy (CIDP)

Developmental disorders (cerebral palsy, Arnold-Chiari malformations)

Dysautonomic states

Encephalitis

Facioscapulohumural dystrophy (FSHD)

Granulomatous disorders (neurosarcoidosis, Wegener's granulomatosis)

Hypothalamic and pituitary diseases

Intracranial infections (meningitis and encephalitis)

Metabolic encephalopathy and mitochondrial diseases

Migraine

Motor neuron disease

Multiple sclerosis

Multiple system atrophy

Myotonic dystrophy

Narcolepsy and related sleep disorders

Paraneoplastic (limbic encephalitis, opsoclonus-myoclonus)

Parkinson's disease and other parkinsonian disorders

Pompe disease and other metabolic myopathies

Cerebral commotion

Guillain-Barré syndrome (GBS)

Post-infection fatigue states (poliomyelitis, Lyme disease, Q-fever, and viral infections)

Postoperative (posterior fossa) surgery

Adapted from Ref. [14]

(GBS), an immune-mediated polyradiculoneuropathy, and Pompe disease, a metabolic muscle disorder. Various aspects of fatigue will be discussed, including differences between experienced and physiological fatigue and different methods available for assessment of fatigue. GBS and Pompe disease are taken as examples to discuss possible pathological mechanisms of fatigue, treatment strategies and options for future research.

\section{Different types of fatigue and their definitions}

Fatigue covers a broad spectrum of symptoms and complaints, and has no uniform definition. In clinical research, commonly used definitions of fatigue are an overwhelming and persistent feeling of tiredness and diminished ability to sustain voluntary mental and physical activities. In addition, weakness, lethargy and lack of energy that interfere with daily activities are used as definitions [9, 13, 22, 23,
$29,51,53,57,107]$. In basic neurosciences, it is defined as a time-related force decline [32, 102]. Considering the different aspects of fatigue it is best regarded as a multidimensional concept in which the level of experienced fatigue and the ability to perform activities are influenced by the type of disease, the health status of the patient and several aspects of patient functioning. A distinction is made between experienced fatigue and physiological fatigue, which can both be influenced by psycho-sociological factors [101]. The multidimensional concept of fatigue is depicted in Fig. 1. In this figure fatigue is integrated in the World Health Organization's International Classification of Functioning, Disability and Health (WHO-ICF). The WHO-ICF represents the effect of disease on body function and structure and the level of activities and participation of the patient [1].

\section{Physiology of fatigue}

With electrophysiological testing, fatigue can be measured as a loss of voluntary force-producing capacity of the muscles during exercise $[8,72]$. To understand this reduced capacity of the muscle it is important to know how fatigue arises. The central governor model clarifies in a logical way how fatigue arises during mental or physical effort and how fatigue is essential for protecting the body against damage due to excessive exercise [69]. During performed effort the CNS is continuously informed about the level of perceived exertion by feedback from muscles, joints, body temperature, the cardiorespiratory system and cognitive domains (afferent pathways). This feedback is processed at the primary somatic sensory cortex, subsequently sensory motor integration takes place and the activation by the primary motor cortex of the brainstem motor nuclei and anterior horn cells in the spinal cord (efferent pathway) is adjusted if needed to maintain body homeostasis. This continuous feed forward and feedback control mechanisms regulate the work rate and determines when rest is needed [14, 69].

With this model it is not fully explained how severe fatigue can be present in chronic disease or how fatigue can be present in a resting situation. In patients with severe fatigue the sense of normal fatigue is amplified due to pathological changes in the motor system, disruption of feedback to the primary somatic sensory cortex and/or change in patients' motivation [14, 69]. In a resting situation an estimation is made whether the body can cope with an increase in effort. Up-regulation of metabolism occurs during exercise; however, in case of disease, the capacity to increase the metabolic rate is limited. This is perceived at the level of the CNS as fatigue and the patient will avoid excessive effort and requires rest. 


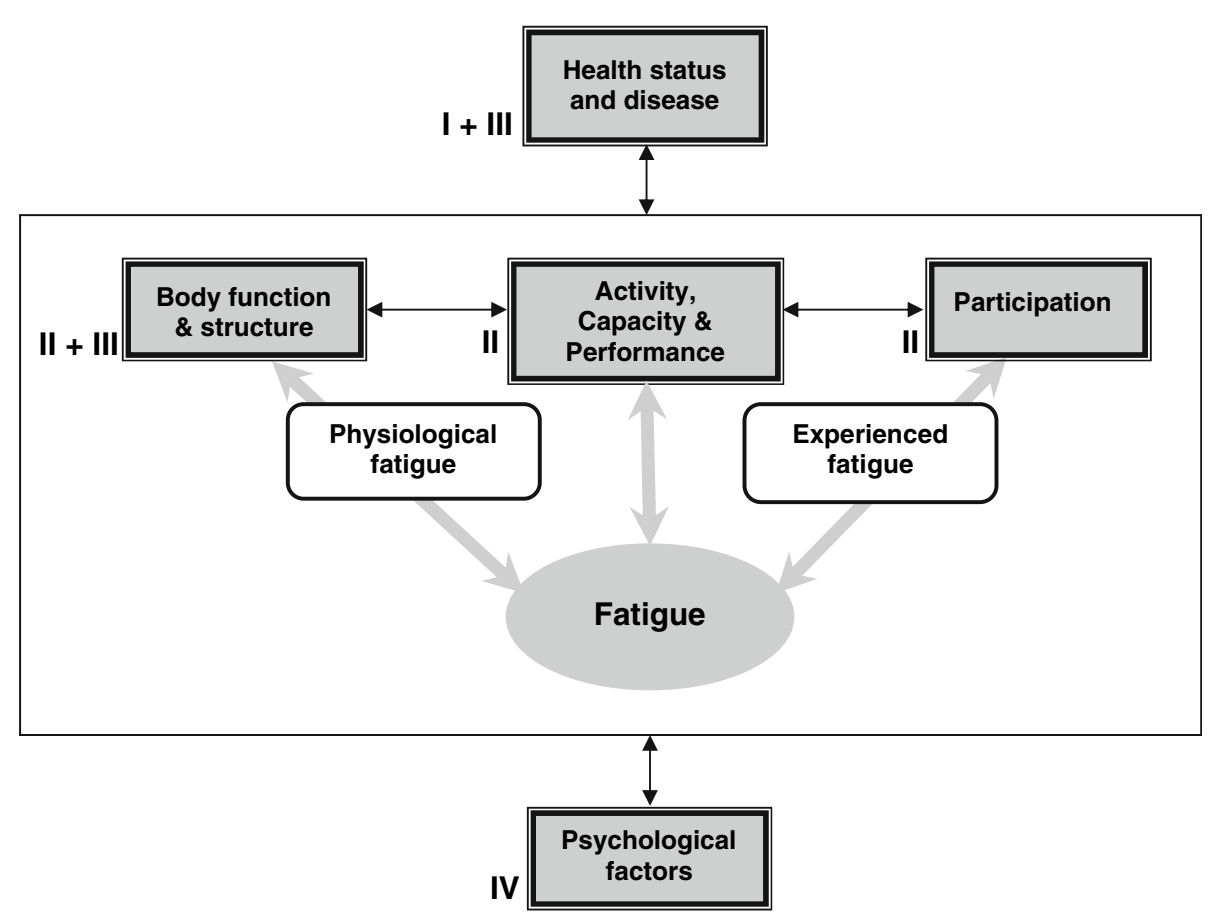

Fig. 1 Fatigue as a multidimensional concept implemented in the World Health Organization's Classification of Functioning, Disability, and Health. The multidimensional concept of fatigue is integrated in the World Health Organization's International Classification of Functioning, Disability and Health (WHO-ICF), representing the effect of disease on body function and structure, activity and participation of the patient [1]. Both experienced fatigue and

In systemic diseases loss of voluntary force-producing capacity is caused by multisystemic consequences of the underlying disease. In neurological disease a distinction can be made between fatigue caused by disease at the level of the CNS (central fatigue) versus fatigue caused by disease at the level of the PNS (peripheral fatigue). Neurological diseases can be located at various levels of the nervous system, such as the motor cortex, the spinal cord, the peripheral nerve, the neuromuscular junction or the muscle itself [107]. Most of these structures are either important feedback pathways for the perceived effort or important in voluntary force-producing capacity of the muscle. Presumably for that reason, severe fatigue is commonly seen in a variety of neurological diseases.

\section{Central fatigue}

Central fatigue is the term generally used when symptoms result from either psychiatric disease or pathology in the CNS. This can result in either difficulty with sustaining physical activities and/or impaired cognitive functioning. For example, patients with structural lesions in brain areas important for cognitive processing will have limited ability to sustain concentration and endure mental tasks (mental fatigue) $[5,11,13,14]$. Psychiatric diseases may disturb physiological fatigue have an effect on activity and participation and are in most diseases related to health status and disease severity. Psychosocial factors have an influence on fatigue and on activity and participation. The numbers indicate at which level the different treatment strategies have an effect on fatigue: $I$ treatment of underlying disease; II rehabilitation and exercise; III pharmacotherapeutics; $I V$ cognitive behavior therapy

neuronal processing in the CNS, for example, in depression there is reduced internal input from the CNS, which results in loss of interest and motivation and an exaggerated sense of fatigue [14]. Loss of voluntary force-producing capacity of the muscles during exercise due to central mechanisms seems to result from suboptimal input from the CNS. This is observed in patients with diseases disrupting the motor cortex or the motor pathways of the CNS such as multiple sclerosis [84]. Endocrine disturbances as seen in hypothalamic-pituitary diseases may give rise to profound chronic fatigue [14]. Temporal hypothalamic-pituitary dysregulation is also responsible for fatigue as seen in the overtrained athlete syndrome [3].

\section{Peripheral fatigue}

Peripheral fatigue, which is similar to muscle fatigability, is likely to occur in a patient showing a topographic pattern of muscle weakness or sensory disturbances at neurological examination based on dysfunction of the lower motor unit or sensory nerves. Patients with metabolic myopathies (e.g., Pompe disease), with disorders at the level of neuromuscular transmission (e.g., myasthenia gravis), with disorders affecting the peripheral nerves [e.g., GBS and Charcot Marie Tooth type 1 (CMT type 1)] and the anterior horn 
cells (e.g., motor neuron disease) can all suffer from peripheral muscle fatigability, which is characterized by failure to sustain the force of muscle contraction over time. In patients with myopathies, muscle fatigability is enhanced due to structural changes or altered muscle metabolism in the muscle, which hampers muscle contraction. When motor conduction is impaired, this leads to decreased recruitment of motor units, resulting in an earlier occurrence of muscle fatigability [14]. Myasthenia gravis is characterized by rapid progressive reduction of muscle strength with repeated muscle contraction with (partial) recovery after a period of rest. This phenomenon of inability to sustain physical activities is caused by anti-acetylcholine (Ach) receptor antibodies and a reduction in the number of $\mathrm{ACh}$ receptors of the neuromuscular junction [19].

Fatigue of central origin in neuromuscular disease

Interesting is the finding that patients with PNS disease also suffer from a generalized form of fatigue, which originates at the level of the CNS. This should not be confused with muscle weakness or (exercise induced) muscle fatigability. Previously, it was thought that generalized fatigue was caused by skeletal muscle hypoxia or anaerobiosis; however, these metabolic changes should lead to muscle rigor $[69,73]$. Furthermore, one would expect that the primary motor cortex tries to compensate for the decrease of generated force by recruiting the maximum number of motor units; however, this is not seen during maximal exercise [87]. For example, in muscle disease, if muscle metabolism is beforehand affected, this will result in a continuous afferent feedback to the CNS. The CNS will minimize activities to protect further damage to the muscles (central governor model). In case of muscle weakness an increase in applied effort is needed to perform the same activities as a healthy person. This will lead to a higher perceived exertion at the level of the primary somatic sensory cortex and an earlier occurrence of fatigue $[14,69]$. In neuro-inflammatory diseases, e.g., post-poliomyelitis syndrome or chronic inflammatory demyelinating polyneuropathy (CIDP), fatigue may arise from dysregulation of the neuro-endocrine system. The local inflammatory process in the muscle or nerves may trigger a systemic inflammatory response, which causes neuroendocrine dysregulation.

\section{Experienced fatigue}

Experienced fatigue is the patient's perception of his or her level of fatigue in daily life, which is alike to feeling or sense of fatigue $[14,107]$. This reflects to what extent individual patients notice the increase in effort needed to complete daily activities and their limitations in endurance during sustained physical and mental activities [107]. It is a subjective form of fatigue. The way how fatigue is experienced is influenced by psycho-sociological factors of the patient, such as attributions towards fatigue, coping mechanisms, overall well-being and social circumstances (Fig. 1) [101]. For example, patients who are stress-sensitive or encounter a stressful event in life are often in a fight or flight state, characterized by activation of the sympathetic nervous system and initiation of the acute stress response via the hypothalamic-pituitary-adrenal axis. These prolonged stress responses may result in exhaustion and feelings of fatigue. Certain individual patient characteristics, such as emotional state and lack of motivation, may cause a dissociation between the level of internal input (motivational and limbic) and that of the perceived signal of performed effort [14]. This dissociation is responsible for an increased perception of fatigue. Experienced fatigue and physiological fatigue can be related to each other, but do not necessarily co-occur within individuals. In CMT type 1, facioscapulohumeral dystrophy (FSHD) and myotonic dystrophy, central fatigue (not peripheral fatigue) correlates with experienced fatigue; however, in GBS no correlation has been found between either peripheral or central fatigue $[35,81]$.

\section{How to assess fatigue}

The broad range of mechanisms underlying fatigue, its multi-dimensional character, confounding factors and different manifestations of fatigue put high demands on the way fatigue is measured. It is often difficult to assess the difference in severity of fatigue among individuals. Nevertheless, quantification of fatigue and its impact on the patient's life are important for good insight in the overall condition of the patient and may be helpful in deciding which supportive measures should be taken to alleviate fatigue [72].

Several methods have been developed to assess the level of fatigue. These measurements can be divided into two categories. The first one, generally based on patientreported outcome measures, focuses on experienced fatigue. The second category is based on objective tests and outcomes, and focuses on physiological fatigue [22, 58]. The different techniques quantifying the severity of each form of fatigue will be discussed in the next paragraphs.

\section{Ways to measure experienced fatigue}

Experienced fatigue needs to be quantified via patientreported outcome measures (questionnaires). Most of these 
questionnaires are designed for use in daily practice, can be completed in a limited amount of time and provide the physician with an estimate of the severity of fatigue and its impact on the patients' daily life. To measure treatment effects or fluctuations in severity of the underlying disease, a scale should be responsive to changes over time [22]. A distinction is made between unidimensional and multidimensional scales. A unidimensional scale approaches fatigue as a single construct, while multidimensional scales assess several aspects of fatigue, such as physical, cognitive and psychosocial aspects. An elaborate review by Dittner et al. [22] gives a systematic overview of 10 unidimensional and 20 multidimensional scales for the measurement of fatigue. It also provides guidance on choosing the appropriate scale for a particular patient population.

An example of a unidimensional fatigue scale frequently used in neuromuscular disorders is the Fatigue Severity Scale (FSS). This test is easy to use in clinical practice, as the nine items of the test can be answered within less than 5 min. The FSS measures fatigue by assessing the consequences of fatigue on daily functioning. The scores of the scale can range from 1 (no fatigue) to 7 (extremely fatigued). An average score of 4 and higher is indicative for fatigue, and a score of 5 and higher for severe fatigue [27, $40,45,56,64]$. The FSS demonstrated good internal consistency (Cronbach's alpha coefficient $=0.88$ ), test-retest reliability (Cohen's kappa value $=0.84$ ) and discriminative validity in studies among patients with immunemediated polyneuropathies, multiple sclerosis and Pompe disease $[22,38,40,56]$.

An example of a multidimensional fatigue scale is the Checklist Individual Strength (CIS). This is a 20-item questionnaire that evaluates fatigue severity in general (i.e., not related to specific daily activities), concentration, motivation and the level of physical activity [81]. The CIS was not specifically validated for patients with neuromuscular disorders, but has shown good psychometric properties in other populations (internal consistency; Cronbach's alpha coefficient $=0.90$ ) and discriminative validity between multiple sclerosis and healthy controls) [7, 89, 97]. The CIS has been used to assess fatigue in patients with chronic fatigue syndrome, multiple sclerosis, FSHD, CMT type 1 and myotonic dystrophy [81, 83, 89, 97, 101].

\section{Ways to measure physiological fatigue}

There is limited experience with methods that have the potential to objectively quantify the level of physiological fatigue. The following methods can be used: electrophysiological measurement of decline in muscle force after exercise (peripheral fatigue); electrophysiological measurement of central activation failure (central fatigue) [107] and; neuropsychological assessment (central fatigue) [14].

The most direct method for measuring peripheral fatigue is measuring the generated force by electrical stimulation during rest and after muscle contraction and to look at the decline in generated force (Fig. 2). The decline in force reflects the severity of fatigue. It also shows the change in muscle contractibility, such as a fatigue-induced delay of the muscle relaxation phase (Fig. 2) [18]. During the contraction a decline over time of the maximal voluntary force can be observed (muscle fatigability). The actual force exerted is usually expressed as a percentage of the maximal force (e.g., $70 \%$ of the maximal voluntary contraction). With a surface electromyogram a decline in fiber conduction velocity may be indicative for peripheral fatigue [107].

When central fatigue is present, suboptimal input from the CNS to activate the muscle results in a decrease in maximal force generating capacity of the muscle. This is defined as central activation failure (CAF). When during maximal voluntary contraction the generated force increases with electrical stimulation, this is indicative for CAF (Fig. 2). When CAF increases during exercise, this is supportive for the presence of central fatigue. CAF can be confirmed with a twitch-interpolation technique, magnetic and electrical stimulation of the motor cortex and readiness potential. For a commentary on these methods, the review by Zwarts et al. is recommended [107].

A method to evaluate mental fatigue is through neuropsychological assessment. Via this assessment cognitive

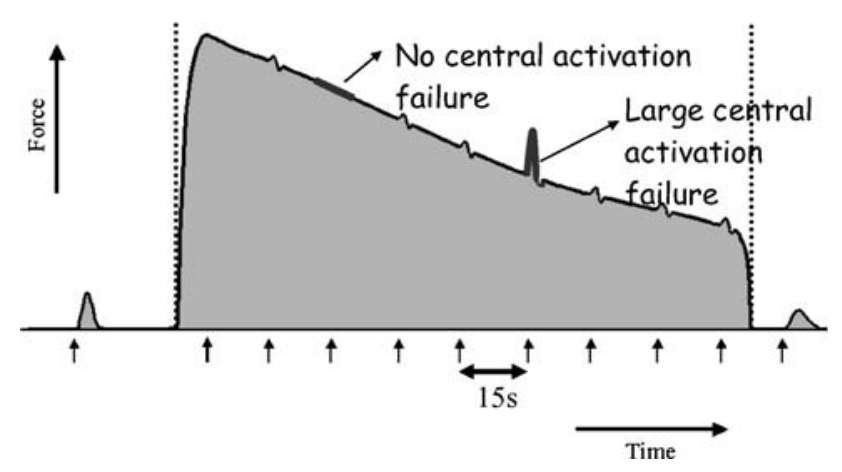

Fig. 2 Schematic representation of physiological fatigue. The figure shows the decline over time of the maximal voluntary force (on the Y-axis). The 'at-rest twitches' are visible before and after the contraction, with the post-contraction twitch being clearly lower and slower, indicative of peripheral fatigue. The arrows indicate the moments of superimposed electrical endplate stimulation. The twitch interpolation has induced small increments in muscle force with examples of a negligible and a large central activation failure (CAF). A (near) absent response indicates a full voluntary activation of the muscle. This figure originates from Ref. [107] 
domains of memory, learning, attention and information processing are evaluated. Patients with mental fatigue perform worse on these tests, and their performance will decline during the assessment session $[5,11]$.

\section{Fatigue in neuromuscular disorders}

Neuromuscular disorders include a wide range of disease entities causing abnormalities in muscle function and/or sensory function. These may be caused by dysfunction at the level of the muscle itself, the neuromuscular junction, the peripheral nerve or at the level of the anterior horn cell in the spinal cord or brain stem. Typical features of neuromuscular disorders comprise muscular atrophy, muscle weakness and sensory disturbances such as pain, tinglingfeelings, hyperpathy or hypoesthesia [19].

Fatigue has been an under-recognized aspect of neuromuscular disorders over a long period of time. As we have mentioned before, fatigue on one hand is caused by a local effect on muscle function (peripheral fatigue), and on the other hand fatigue originates at the level of the CNS because of feedback about the pathological state of the PNS. This fatigue protects the muscles from further damage by down-regulating physical activities. This is illustrated by the fact that fatigue of central origin was recorded in $36-41 \%$ of patients with a neuromuscular disorder (FSHD, CMT type 1 and myotonic dystrophy) vs. $12 \%$ in controls [82]. These high percentages, however, were not found in a small study in GBS patients [35]. In patients with FSHD, CMT type 1 and myotonic dystrophy, fatigue of central origin correlated positively with the level of experienced fatigue. In GBS patients, this correlation has not been found $[35,81]$. It should be noted that most individuals with severe fatigue, but without demonstrable muscle weakness and/or elevated serum creatine kinase (CK) levels do not have a neuromuscular disorder. Thus, fatigue as the only complaint excludes in most cases the presence of a neuromuscular disorder [59]. An exception to this rule are mitochondrial myopathies or other metabolic disorders in which clear exercise intolerance can reported by patients where muscle weakness is not always present at the time of the neurological examination [14]. Furthermore, fatigue can persist as a residual symptom after a neurological illness, of which GBS and CIDP are good examples [38, 64].

Other disorders in which increased levels of fatigue have been reported are myasthenia gravis, post-poliomyelitis syndrome, immune-mediated neuropathies, FSHD, CMT type I, myotonic dystrophy and Pompe disease [38, 40, 43, $48,53,64,71,78,81]$. The prevalence of fatigue in various neuromuscular disorders reported in the literature ranges from 38 to $86 \%$ (Table 2). The reported prevalence of fatigue in neuromuscular disorders compares with the prevalences of fatigue in multiple sclerosis (range from 53 to $92 \%$ ) [10], where fatigue is recognized as an important symptom [54]. To be aware of the impact of fatigue in neuromuscular disease, it is valuable to compare its high prevalence with the low prevalence of severe fatigue in healthy individuals (range 5-18\% and mean FSS 2.3-3.3) $[64,78,92]$. The ranges seen in the mentioned prevalences are explained by differences in study population, used, assessment method and used definition of severe fatigue (e.g., cutoff point).

In the following section of this review we will discuss two different neuromuscular disorders for which fatigue has been reported as an important feature. GBS will be taken as an example of immune-mediated polyneuropathies and; Pompe disease as an example of metabolic myopathies.

\section{Fatigue after Guillain-Barré syndrome (GBS)}

GBS is an acute post-infectious immune-mediated polyneuropathy, characterized by rapidly progressive, symmetrical limb weakness and areflexia. Sensory disturbances, autonomic dysfunction and respiratory insufficiency often occur during its disease course. The severity of
Table 2 Prevalences of severe fatigue in neuromuscular disorders

CIS checklist individual strength, FIS fatigue impact scale, FSS fatigue severity scale, $M F I$ multidimensional fatigue inventory, VAS-F visual analogue scale for fatigue, $N A$ not applicable

\begin{tabular}{lll}
\hline Neuromuscular disorder & $\begin{array}{l}\text { Prevalence } \\
\text { of severe } \\
\text { fatigue }(\%)\end{array}$ & $\begin{array}{l}\text { Fatigue scale } \\
\text { used to assess } \\
\text { severity of fatigue }\end{array}$ \\
\hline Guillain-Barré syndrome [28, 38, 64, 78] & $38-86$ & FSS, FIS, VAS-F \\
Pompe disease [40] & 67 & FSS \\
Myasthenia gravis [71] & 82 & FIS, MFI \\
Post-poliomyelitis syndrome [53] & 80 & NA \\
Facioscapulohumural dystrophy [48, 81] & $51-61$ & CIS \\
Myotonic dystrophy [48, 81] & $53-74$ & CIS \\
Charcot-Marie-Tooth disease type 1 (CMT type 1) [48, 81] & $55-64$ & CIS
\end{tabular}


neurological deficits differs among patients and usually reaches a plateau phase within 2 weeks, which is indicative of the start of the recovery phase. Final outcome varies greatly among patients and ranges from severe residual weakness to complete recovery $[4,99]$. CIDP may be considered a chronic variety of GBS [98].

Patients experience fatigue already at the onset of GBS, and it can surprisingly persist for many years, even after total recovery of muscle weakness [38]. Fatigue in GBS was sporadically reported in the literature until 1999, when a cross-sectional case control study showed that fatigue is an essential and incapacitating residual symptom in patients with immune-mediated polyneuropathies [64]. Eighty percent of patients that recovered from GBS or CIDP or had ongoing CIDP suffered from severe fatigue. The patients stated that being severely fatigued leads to tremendous impairment in their daily life and social activities. As a consequence their quality of life was reported to be negatively affected in the long term. Some confounding factors could have affected the outcome of the study addressing fatigue in GBS. Sleep disorders, depression and level of physical activity were not systematically evaluated in the study. However, other studies confirmed the high prevalence of fatigue in GBS, being 38\% [78], $40 \%$ [28] and 86\% [64]. Part of the differences in estimated prevalence can probably be explained by differences in age between study populations, as severe fatigue showed a positive correlation with age in GBS [38]. Severe fatigue does not seem to be related to clinical variables, antecedent infections or the severity of the neurological dysfunction in the initial phase of GBS $[38,64]$, except that severe fatigue has a higher prevalence in female GBS patients $(74 \%$ in females versus $45 \%$ in males, $p=0.003$ ). This difference in sex ratio is also seen in patients with chronic fatigue syndrome and can probably best be explained by endocrine differences between males and females [77]. A conventional nerve conduction study failed to show a relation between experienced fatigue and the occurrence of physiological fatigue/residual nerve dysfunction [37]. However, it could well be that newer or more advanced electrophysiological methods could be helpful to study this relationship. It has been added that CMAP scans potentially could be helpful to study this relationship [24].

Another interesting finding was that severely fatigued GBS and CIDP patients showed a normal activity level, which was independent of their neurological condition. We presume that this neglect of patients' impaired load capacity may even increase symptoms of fatigue, as the actual performance of the patient does not match the patient's physical load capacity [12]. GBS not only affects the peripheral nerves, but also has an impact on psychosocial status. This may lead to a disturbed interplay between the structural and/or functional integrity of sensory, motor and cognitive symptoms, which may increase the sense of fatigue.

In general, the occurrence of fatigue in GBS seems independent of the severity of (residual) neurological deficits [37, 38, 64]. This differs from findings in other neuromuscular disorders, where severely affected patients experience higher levels of fatigue [40, 49]. The fact that GBS is an acute and monophasic disease may explain at least part of the differences found, as the other mentioned neuromuscular disorders are often chronic diseases and progressive in nature. In GBS and in CIDP it could well be that dysregulation of the hypothalamic-pituitary-adrenal axis accounts for residual fatigue, which could be linked to the antecedent infections seen in GBS and smoldering low-grade infections possibly going on in CIDP, because similar immune-mediated fatigue is seen as a residual symptom after an infectious illness [46]. Dysautonomia could also be an important contributor to fatigue [14] and is frequently seen in patients with GBS. At the moment further prospective studies in GBS are being executed (as part of the Dutch 'Graph study') to investigate the relation between fatigue in relation to dysautonomia, preceding infections, severity of disease and the occurrence of pain (L. Ruts, personal communication). In conclusion, we could state that peripheral fatigue is a more important contributor in the initial phase of the GBS. However, because feelings of fatigue persist after resolution of neurological symptoms, other factors must be responsible for fatigue in the long run. Post-infectious fatigue, dysautonomia and psycho-sociological consequences of the disease may be these causative factors.

\section{Fatigue in Pompe disease}

Pompe disease (glycogen storage disease type II, acid maltase deficiency) is an autosomal recessive disorder caused by deficiency of lysosomal acid alpha-glucosidase. As a result, glycogen accumulates in lysosomes of several body tissues [52]. The disease presents as a spectrum of phenotypes. Patients with the classic infantile form are at the severe end of the spectrum. They suffer from a rapidly progressive disease course. Presentation is shortly after birth with hypotonia, feeding difficulties or respiratory problems. A hypertrophic cardiomyopathy is characteristically present. Patients usually die before the first year of life because of cardiorespiratory failure [94]. On the other end of the spectrum are children and adults with Pompe disease in which skeletal muscles are primarily affected. In these patients the disease is slowly progressive. The proximal, paraspinal and respiratory muscles are most involved. Patients may eventually become wheelchair bound and dependent on (nocturnal) ventilation [42, 43]. 
Fatigue is a frequently experienced symptom in adults with Pompe disease and may have a disabling impact on the lives of patients [40]. Until recently, fatigue in Pompe disease did not receive much attention and was not structurally assessed. Incidentally fatigue had been reported in case reports and small patient populations of up to 16 subjects $[20,62,65,85,105]$. In a study on the natural course of Pompe disease in 54 patients, fatigue was rather unexpectedly reported to be the presenting symptom in $25 \%$ of the patients [43]. Seventy-six percent of the study population indicated that fatigue had an important impact on their daily life. Subsequently, fatigue was studied by using the FSS in more detail in an international group of 225 patients [40]. It was demonstrated that fatigue occurs in the majority $(78 \%)$ of Pompe patients (FSS score $\geq 4$ ) and severe fatigue (FSS score $\geq 5$ ) in two-third of the patients [40]. The mean FSS scores differed significantly from healthy controls (mean FSS score of 5.2 versus 2.9, $p<0.001)$. Patients who did not use a wheelchair and respiratory support were significantly less fatigued when compared with patients using either or both $(p=0.01)$ [40]. It is of interest that Pompe patients reported only slightly lower levels of cognitive well-being in comparison with the general population [41]. This indicates that depression probably does not contribute to the observed prevalence of experienced fatigue, in contrast to, for example, multiple sclerosis, for which depression was found to have a substantial influence on the severity of fatigue [74].

In Pompe disease the accumulation of glycogen in muscle fibres leads to structural changes of the muscle, which impairs muscle contraction, and in a more advanced state even destructs muscle fibers. This results in muscle weakness, muscle atrophy and muscle fatigability (peripheral fatigue). When patients are performing physical activities, they experience burning sensations of the proximal muscles and a heavy-legged feeling, which limits their force-producing capacity. In Pompe disease and other metabolic myopathies, such as McArdle's disease, muscle metabolism is altered, and certain metabolites could impair skeletal muscle contraction through a direct action. This would be perceived as an increase in effort by the CNS, which then responds with down-regulating of activities to preserve homeostasis in the muscle [69].

Respiratory dysfunction, due to diaphragmatic weakness and weakness of the respiratory muscles, is often present in Pompe patients and can also account for an increase in fatigue of central origin. Patients have to increase their respiratory effort to sustain adequate ventilation during exercise, or even already at rest. By feedback from the respiratory system (hypercapnia and hypoxia), fatigue is perceived at the CNS, and adequate ventilation is restored by decreasing the performed physical effort [14, 69].
Second, nocturnal hypoventilation leads to a decreased oxygenation of the brain and respiratory acidosis. Due to altered respiratory function the normal sleep pattern is disrupted [63]; as a consequence patients are not well rested, feel fatigued and suffer from day-time sleepiness during the day.

The pathophysiology of fatigue in Pompe disease still remains to be established, as this topic has not yet been investigated. Muscle fatigability of the proximal muscles plays an important role in Pompe patients, as they have difficulties in sustaining physical activities. Besides this localized form of fatigue, the occurrence of generalized fatigue is explained by the central governor model [14, 69]. Respiratory dysfunction also contributes to the level of fatigue perceived at the CNS.

\section{Implications for treatment}

Fatigue is a complex and multifaceted entity. In order to determine the treatment strategy for an individual patient, it is important to define the type of fatigue and the underlying factors that contribute. First, the implications of fatigue for the patient need to be investigated. This can be done by assessing the level of fatigue and its impact on daily activities and general well being. Second, potential behavioral and psycho-sociological factors need to be identified that contribute to the experienced level of fatigue. Currently one or more of the following optional treatment strategies for fatigue in neuromuscular disorders can be used: (1) (symptomatic) treatment of the underlying disease, (2) rehabilitation and exercise, (3) pharmacotherapeutics directed to treat fatigue and (4) cognitive therapy. Figure 1 shows how the different treatment strategies act on fatigue and the different domains of the WHO-ICF model.

\section{Treatment of underlying disease and symptoms}

When a patient complains of symptoms of fatigue, a diagnostic workup should be done to identify potential causes of fatigue. As mentioned, fatigue can be a symptom of various (neurological) diseases, but also other underlying diseases may cause fatigue [14]. When the patient has already been diagnosed with a disorder that may explain fatigue, other causes like co-morbidity need to be ruled out. When possible, start or optimize treatment of the underlying disease, and eliminate other causal factors that may contribute to fatigue. For example, treatment of myasthenia gravis patients with adequate dosages of pyridostigmine can improve the clinical well-being and reduce feelings of fatigue $[39,47]$. In a pilot study of late-onset Pompe 
patients treated with enzyme replacement therapy, the patients experienced a reduction in fatigue over a period of 8 years of treatment [93]. The experienced increase in energy level made them more independent in daily life and improved their participation in social activities.

Furthermore, in patients with neuromuscular disorders it is of utmost importance to rule out sleep-disordered breathing as a cause of sleepiness and fatigue during the day [76]. Nocturnal hypoventilation is frequently seen in neuromuscular patients with diaphragmatic weakness or in patients with obstructive apnoea or hypopnea. Besides hypoventilation, obstructive sleep apnea causes a disrupted sleep pattern, which results in a lowered quality of sleep [86]. Symptoms explained by nocturnal hypoventilation often herald complete respiratory failure by months or even years. Diaphragmatic weakness is often seen in Pompe disease and is present when there is a significant decline in pulmonary function when pulmonary function is measured in the sitting and the supine position [96]. A decline of $25 \%$ or more is called postural drop. Sleep-disordered breathing can be measured with sleep studies. When mechanical ventilation is implemented in patients suffering from nocturnal hypoventilation or sleep disordered breathing, symptoms of fatigue can be tremendously reduced [76].

\section{Rehabilitation and exercise}

A well-fitted rehabilitation program can help patients to use their energy more efficiently. For example, implementation of ergo-therapeutic devices aimed at improving the ease of performing daily activities may increase the level of energy and thereby lessen symptoms of fatigue. Also training in developing coping strategies aimed at balancing mental and physical activities and rest may improve the patient's well- being. The potential effect of exercise programs on the level of fatigue was investigated in several studies $[12,25,34$, 79]. In patients recovered from GBS and in patients with ongoing but well-treated CIDP who suffer from severe fatigue, implementation of tailor-made exercise programs resulted in a reduction of $20 \%$ of self-reported fatigue. This improvement had been maintained at 2-year follow-up [33, 34]. These findings are consistent with results reported for healthy sedentary individuals $(15 \%)$ and patients with multiple sclerosis (22\%) after completion of an exercise program $[75,80]$. However, the decrease in experienced fatigue in GBS after exercise therapy could not be explained by an increase in physical fitness [12]. Hypothetically, it could be that training also has a beneficial effect on the neuro-endocrine system and levels of neurotransmitters, which may reduce perception of fatigue. Additionally, recognition of the patients' complaints of fatigue and the beneficial effect of support from fellow patients within the training group are also potential factors that can reduce experienced fatigue $[12,34]$. Figure $3 \mathrm{a}, \mathrm{b}$ visualizes the relationships among training, physical fitness, activity and psychosocial factors in a model. Figure 3a shows the relationships as was hypothesized before the start of the training study and Figure $3 \mathrm{~b}$ the remodeling after analysis of the results of the study [12,34].

Although strong evidence for a beneficial effect of exercise on experienced fatigue in neuromuscular disorder presently is relatively scarce, a low-intensity training schedule is likely to have a positive effect on fatigue and the general condition of patients with a neuromuscular disorder. Regular activity prevents physical deconditioning and muscle wasting. This was also shown in a rat model for post-poliomyelitis where physical activity reduced the size of the chronically enlarged motor units in denervated muscles [91].
Fig. 3 Model of fatigue in immune-mediated polyneuropathies. a Represents the hypothetical model of the mechanisms between the different domains in relation to physical training. b Is the model resulting from the correlations between the change scores of the different domains as a result of the training intervention. Thickness of lines between domains represents the strength of the relationship. Figures adapted from Ref. [12]
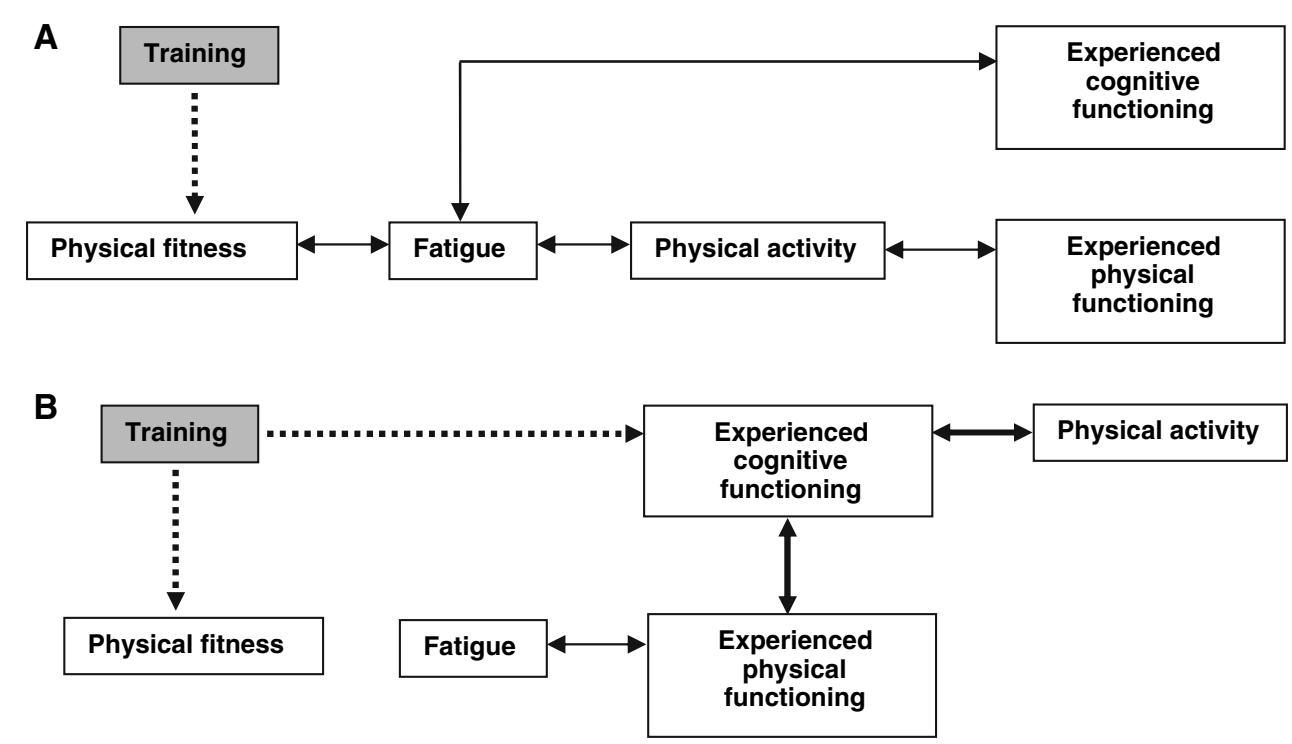


\section{Pharmacotherapeutics}

The efficacy of pharmacotherapeutic drugs such as amantadine, modafinil, albuterol and aminopyridine has not yet been convincingly proven for the treatment of fatigue in patients with neuromuscular disorders. Amantadine was shown to be effective in one-third to half of all treated multiple sclerosis patients [15, 55, 67]. Amantadine, however, did not show a beneficial effect in a well-powered randomized controlled trial on residual fatigue in patients who were in a good clinical condition after recovery from GBS [36]. In FSHD the effects of albuterol had no effect on experienced fatigue [95]. Modafinil has shown some beneficial effect in myotonic dystrophy; however, these were studies with small populations and a relatively short follow-up [17, 61, 90, 103]. Clinical trials studying the efficacy of pharmacotherapeutics as treatment for fatigue in other neuromuscular disorders are still lacking.

\section{Cognitive behavior therapy}

Patients' attitudes towards fatigue, coping mechanisms and psychosocial circumstances attribute to the level of experienced fatigue [101]. Patients may experience stress, depression and anxiety caused by disease-related social consequences, such as loss of employment, failure to fulfill family responsibilities and limitations in their social life. If necessary, patients can be supported with occupational rehabilitation. Cognitive behavioral therapy and psychological support have been shown to have a positive effect on experienced fatigue in cancer patients [68]. Whether the same results can be obtained in patients with neuromuscular disorders needs further investigation.

\section{Conclusion and future lines of investigation}

The impact of fatigue on the lives of patients suffering from neuromuscular disorders has remained long unnoticed and has only recently been recognized as an important subject to be addressed in both clinical practice and research [6, 14, 38, 40, 64, 104, 107]. A high prevalence of fatigue was found in Pompe disease, GBS, post-poliomyelitis, hereditary polyneuropathy, myasthenia gravis and myotonic dystrophy [38, 40, 48, 53, 64, 70]. The prevalence of severe fatigue in these disorders was estimated to be similar to that in patients with multiple sclerosis [50].

Fatigue can be subdivided into experienced fatigue and physiological fatigue. The severity of both experienced fatigue and physiological fatigue is influenced by psychosociological factors. In most neuromuscular disorders, but not in GBS as far as we know now, it also depends on disease severity [38, 40, 49, 64, 100]. The pathophysiologic mechanisms causing fatigue in neuromuscular disorders are not completely understood. Most likely there are two main pathways that lead to physiological fatigue: first, the pathology located in the PNS causes muscle fatigability by hampering muscle contraction, disturbance of muscle metabolism and inability to activate enough motor units (peripheral fatigue). Because patients with neuromuscular diseases have to compensate for their loss of function by increasing their motor input to accomplish the same activities as a healthy person, the perceived level of effort at the CNS is higher, and patients become more easily fatigued. Additionally, the disease state of the muscles is continuously sensed at the level of the CNS, which will result in a constant feeling of fatigue of central origin, because body homeostasis needs to be maintained. Besides this flexible interactive feedback and feed forward system as described in the central governor model, other causes of central fatigue are proposed in patients with neuromuscular disorders [81]. Pathology of the PNS can eventually induce functional changes in the motor cortex, which may be a consequence of deconditioning and/or the relatively large demands put on the affected neuromuscular system [16, 21, 60]. This indirectly results in central fatigue due to CAF. Second, there is some evidence that pathology in neuromuscular disorders is not always restricted to the PNS and can affect the CNS as well. In FSHD and myotonic dystrophy, this was revealed using magnetic resonance imaging and somatosensory-evoked potentials [26, 66]. Although central fatigue usually correlates positively with the level of experienced fatigue, this correlation was not clearly found in 'neurologically well-recovered' GBS patients $[35,81]$.

The differences of several aspects of fatigue found in GBS, as compared to other neuromuscular disorders, may lie in the fact that GBS is an acute and monophasic disease, often preceded by an infection. In contrast, the others neuromuscular diseases are often chronic and mostly progressive diseases.

For the management of fatigue in neuromuscular disorders, it is advised to start with optimizing treatment of the underlying disease and of possible co-existing causes of fatigue. When symptoms of fatigue persist, nonpharmacological interventions are preferable to pharmacotherapeutics, as pharmacotherapeutics have not yet been proven to be effective for the treatment of fatigue in neuromuscular disorders [36]. Further elucidation of the pathophysiology of fatigue, with special interest in the central governor model, may help to guide the development and proper targeting of new and hopefully effective pharmacotherapeutics. Meanwhile, the benefit of nonpharmacological treatments, such as low-intensity physical training, rehabilitation and cognitive behavioral therapy, as 
treatment options for fatigue in neuromuscular disorders needs further evaluation. The increasing awareness of fatigue as an important part of the burden of disease in neuromuscular disorders is not only important for the development of new treatment strategies, but also for improvement of current patient care.

Open Access This article is distributed under the terms of the Creative Commons Attribution Noncommercial License which permits any noncommercial use, distribution, and reproduction in any medium, provided the original author(s) and source are credited.

\section{References}

1. World Health Organization (2001) International Classification of Functioning, Disability and Health: ICF. WHO, Geneva

2. Adams RD (1997) Fatigue, athenia, anxiety and depressive reactions. In: Adams RD, Victor M, Ropper AH (eds) Principles of neurology. McGraw-Hill, New York, pp 497-507

3. Angeli A, Minetto M, Dovio A et al (2004) The overtraining syndrome in athletes: a stress-related disorder. J Endocrinol Invest 27(6):603-612

4. Asbury AK, Cornblath DR (1990) Assessment of current diagnostic criteria for Guillain-Barre syndrome. Ann Neurol 27(Suppl):S21-S24

5. Bagert B, Camplair P, Bourdette D (2002) Cognitive dysfunction in multiple sclerosis: natural history, pathophysiology and management. CNS Drugs 16(7):445-455

6. Bernsen RA, de Jager AE, Schmitz PI et al (1999) Residual physical outcome and daily living 3 to 6 years after GuillainBarre syndrome. Neurology 53(2):409-410

7. Beurskens AJ, Bultmann U, Kant I et al (2000) Fatigue among working people: validity of a questionnaire measure. Occup Environ Med 57(5):353-357

8. Bigland-Ritchie B, Jones DA, Hosking GP et al (1978) Central and peripheral fatigue in sustained maximum voluntary contractions of human quadriceps muscle. Clin Sci Mol Med 54(6):609-614

9. Bleijenberg G (2003) De ene vermoeidheid is de andere niet. Nijmegen. Ref Type: Report

10. Branas P, Jordan R, Fry-Smith A et al (2000) Treatments for fatigue in multiple sclerosis: a rapid and systematic review. Health Technol Assess 4(27):1-61

11. Bruno RL, Creange SJ, Frick NM (1998) Parallels between postpolio fatigue and chronic fatigue syndrome: a common pathophysiology? Am J Med 105(3A):66S-73S

12. Bussmann JB, Garssen MP, Van Doorn PA et al (2007) Analysing the favourable effects of physical exercise: relationships between physical fitness, fatigue and functioning in GuillainBarre syndrome and chronic inflammatory demyelinating polyneuropathy. J Rehabil Med 39(2):121-125

13. Chaudhuri A, Watson WS, Pearn J et al (2000) The symptoms of chronic fatigue syndrome are related to abnormal ion channel function. Med Hypotheses 54(1):59-63

14. Chaudhuri A, Behan PO (2004) Fatigue in neurological disorders. Lancet 363(9413):978-988

15. Cohen RA, Fisher M (1989) Amantadine treatment of fatigue associated with multiple sclerosis. Arch Neurol 46(6):676-680

16. D'Angelo MG, Bresolin N (2003) Report of the 95th European Neuromuscular Centre (ENMC) sponsored international workshop cognitive impairment in neuromuscular disorders, Naarden, The Netherlands, 13-15 July 2001. Neuromuscul Disord 13(1):72-79
17. Damian MS, Gerlach A, Schmidt F et al (2001) Modafinil for excessive daytime sleepiness in myotonic dystrophy. Neurology 56(6):794-796

18. de Ruiter CJ, Jones DA, Sargeant AJ et al (1999) The measurement of force/velocity relationships of fresh and fatigued human adductor pollicis muscle. Eur J Appl Physiol Occup Physiol 80(4):386-393

19. De Visser M, Vermeulen M, Wokke JH (1999) Neuromusculaire ziekten. Elsevier, Maarssen

20. Demey HE, Van Meerbeeck JP, Vandewoude MF et al (1989) Respiratory insufficiency in acid maltase deficiency: the effect of high protein diet. JPEN J Parenter Enteral Nutr 13(3):321-323

21. Di L, V, Oliviero A, Tonali PA et al (2004) Changes in motor cortex excitability in facioscapulohumeral muscular dystrophy. Neuromuscul Disord 14(1):39-45

22. Dittner AJ, Wessely SC, Brown RG (2004) The assessment of fatigue: a practical guide for clinicians and researchers. J Psychosom Res 56(2): 157-170

23. Dobkin BH (2008) Fatigue versus activity-dependent fatigability in patients with central or peripheral motor impairments. Neurorehabil Neural Repair 22(2):105-110

24. Drenthen J, Maathuis EM, Ruts L et al (2008) Serial CMAP scan analysis in Guillain-Barre patients. J Peripher Nerv Syst 13(2): 167

25. Edmonds M, McGuire H, Price J (2004) Exercise therapy for chronic fatigue syndrome. Cochrane Database Syst Rev (3):CD003200

26. Fierro B, Daniele O, Aloisio A et al (1997) Evoked potential study in facio-scapulo-humeral muscular dystrophy. Acta Neurol Scand 95(6):346-350

27. Flachenecker P, Rufer A, Bihler I et al (2003) Fatigue in MS is related to sympathetic vasomotor dysfunction. Neurology 61(6):851-853

28. Forsberg A, Press R, Einarsson U et al (2004) Impairment in Guillain-Barre syndrome during the first 2 years after onset: a prospective study. J Neurol Sci 227(1):131-138

29. Friedman J, Friedman H (1993) Fatigue in Parkinson's disease. Neurology 43(10):2016-2018

30. Friedman JH, Brown RG, Comella C et al (2007) Fatigue in Parkinson's disease: a review. Mov Disord 22(3):297-308

31. Fukuda K, Straus SE, Hickie I et al (1994) The chronic fatigue syndrome: a comprehensive approach to its definition and study. International Chronic Fatigue Syndrome Study Group. Ann Intern Med 121(12):953-959

32. Gandevia SC (1998) Neural control in human muscle fatigue: changes in muscle afferents, motoneurones and motor cortical drive [corrected]. Acta Physiol Scand 162(3):275-283

33. Garssen MP (205) Treatment of Guillain-Barré syndrome and causes and treatment of residual fatigue. Thesis, Erasmus Medical Centre, Rotterdam, pp 93-100 (14-12-2005)

34. Garssen MP, Bussmann JB, Schmitz PI et al (2004) Physical training and fatigue, fitness, and quality of life in Guillain-Barre syndrome and CIDP. Neurology 63(12):2393-2395

35. Garssen MP, Schillings ML, Van Doorn PA et al (2007) Contribution of central and peripheral factors to residual fatigue in Guillain-Barre syndrome. Muscle Nerve 36(1):93-99

36. Garssen MP, Schmitz PI, Merkies IS et al (2006) Amantadine for treatment of fatigue in Guillain-Barre syndrome: a randomised, double blind, placebo controlled, crossover trial. J Neurol Neurosurg Psychiatry 77(1):61-65

37. Garssen MP, Van Doorn PA, Visser GH (2006) Nerve conduction studies in relation to residual fatigue in Guillain-Barre syndrome. J Neurol 253(7):851-856

38. Garssen MP, Van KR, Van Doorn PA (2006) Residual fatigue is independent of antecedent events and disease severity in Guillain-Barre syndrome. J Neurol 253(9):1143-1146 
39. Grob D, Brunner N, Namba T et al (2008) Lifetime course of myasthenia gravis. Muscle Nerve 37(2):141-149

40. Hagemans ML, van Schie SP, Janssens AC et al (2008) Fatigue: an important feature of late-onset Pompe disease. Neurology 254(7):941-945

41. Hagemans ML, Janssens AC, Winkel LP et al (2004) Late-onset Pompe disease primarily affects quality of life in physical health domains. Neurology 63(9):1688-1692

42. Hagemans ML, Winkel LP, Hop WC et al (2005) Disease severity in children and adults with Pompe disease related to age and disease duration. Neurology 64(12):2139-2141

43. Hagemans ML, Winkel LP, Van Doorn PA et al (2005) Clinical manifestation and natural course of late-onset Pompe's disease in 54 Dutch patients. Brain 128(Pt 3):671-677

44. Havlikova E, Rosenberger J, Nagyova I et al (2008) Impact of fatigue on quality of life in patients with Parkinson's disease. Eur J Neurol 15(5):475-480

45. Herlofson K, Larsen JP (2002) Measuring fatigue in patients with Parkinson's disease-the fatigue severity scale. Eur J Neurol 9(6):595-600

46. Hickie I, Davenport T, Wakefield D et al (2006) Post-infective and chronic fatigue syndromes precipitated by viral and nonviral pathogens: prospective cohort study. BMJ 333(7568):575

47. Hughes RA, Donofrio P, Bril V et al (2008) Intravenous immune globulin (10\% caprylate-chromatography purified) for the treatment of chronic inflammatory demyelinating polyradiculoneuropathy (ICE study): a randomised placebo-controlled trial. Lancet Neurol 7(2):136-144

48. Kalkman JS, Schillings ML, van der Werf SP et al (2005) Experienced fatigue in facioscapulohumeral dystrophy, myotonic dystrophy, and HMSN-I. J Neurol Neurosurg Psychiatry 76(10):1406-1409

49. Kalkman JS, Schillings ML, Zwarts MJ et al (2007) The development of a model of fatigue in neuromuscular disorders: a longitudinal study. J Psychosom Res 62(5):571-579

50. Kalkman JS, van der Werf SP, van Engelen BG et al (2002) Fatigue complaints are equally characteristic for neuromuscular diseases as for multiple sclerosis. Neuromuscul Disord 12(7-8): 779. Ref Type: Abstract

51. Karlsen K, Larsen JP, Tandberg E et al (1999) Fatigue in patients with Parkinson's disease. Mov Disord 14(2):237-241

52. Kroos MA (2008) Enzymatic, cellular and molecular aspects. In: Beathmann M, Straub V, Reuser AJ (eds) Pompe disease. UNI-MED, Bremen, pp 18-24

53. Krupp LB (2003) Fatigue. Nutterworth Heinemann, Philadelphia

54. Krupp LB (2003) Fatigue in multiple sclerosis: definition, pathophysiology and treatment. CNS Drugs 17(4):225-234

55. Krupp LB, Coyle PK, Doscher C et al (1995) Fatigue therapy in multiple sclerosis: results of a double-blind, randomized, parallel trial of amantadine, pemoline, and placebo. Neurology 45(11):1956-1961

56. Krupp LB, LaRocca NG, Muir-Nash J et al (1989) The fatigue severity scale. Application to patients with multiple sclerosis and systemic lupus erythematosus. Arch Neurol 46(10):1121-1123

57. Krupp LB, Pollina DA (1996) Mechanisms and management of fatigue in progressive neurological disorders. Curr Opin Neurol 9(6):456-460

58. Krupp U, Duber O, Christ HJ et al (2004) Application of the EBSD technique to describe the initiation and growth behaviour of microstructurally short fatigue cracks in a duplex steel. J Microsc 213(Pt 3):313-320

59. Layzer RB (1993) Muscle pain, cramps and fatigue. In: Engel AG, Franzini-Amstrong C (eds) Myology. McGraw-Hill, New York, pp 1754-1768
60. Liepert J, Schoser BG, Weiller C (2004) Motor excitability in myopathy. Clin Neurophysiol 115(1):85-89

61. MacDonald JR, Hill JD, Tarnopolsky MA (2002) Modafinil reduces excessive somnolence and enhances mood in patients with myotonic dystrophy. Neurology 59(12):1876-1880

62. Matsuishi T, Terasawa K, Yoshida I et al (1982) Vacuolar myopathy with type 2 A fiber atrophy and type 2 B fiber deficiency. A case of childhood form acid alpha-1, 4-glucosidase deficiency. Neuropediatrics 13(4):173-176

63. Mellies U, Stehling F, Dohna-Schwake C et al (2005) Respiratory failure in Pompe disease: treatment with noninvasive ventilation. Neurology 64(8):1465-1467

64. Merkies IS, Schmitz PI, Samijn JP et al (1999) Fatigue in immune-mediated polyneuropathies. European Inflammatory Neuropathy Cause and Treatment (INCAT) Group. Neurology 53(8):1648-1654

65. Mobarhan S, Pintozzi RL, Damle P et al (1990) Treatment of acid maltase deficiency with a diet high in branched-chain amino acids. JPEN J Parenter Enteral Nutr 14(2):210-212

66. Mochizuki H, Hanajima R, Kowa H et al (2001) Somatosensory evoked potential recovery in myotonic dystrophy. Clin Neurophysiol 112(5):793-799

67. Murray TJ (1985) Amantadine therapy for fatigue in multiple sclerosis. Can J Neurol Sci 12(3):251-254

68. Mustian KM, Morrow GR, Carroll JK et al (2007) Integrative nonpharmacologic behavioral interventions for the management of cancer-related fatigue. Oncologist 12(Suppl 1):52-67

69. Noakes TD, St Clair GA, Lambert EV (2004) From catastrophe to complexity: a novel model of integrative central neural regulation of effort and fatigue during exercise in humans. $\mathrm{Br}$ J Sports Med 38(4):511-514

70. Paul RH, Cohen RA, Gilchrist JM (2002) Ratings of subjective mental fatigue relate to cognitive performance in patients with myasthenia gravis. J Clin Neurosci 9(3):243-246

71. Paul RH, Cohen RA, Goldstein JM et al (2000) Fatigue and its impact on patients with myasthenia gravis. Muscle Nerve 23(9):1402-1406

72. Pawlikowska T, Chalder T, Hirsch SR et al (1994) Population based study of fatigue and psychological distress. BMJ 308(6931):763-766

73. Pedersen TH, Nielsen OB, Lamb GD et al (2004) Intracellular acidosis enhances the excitability of working muscle. Science 305(5687):1144-1147

74. Penner IK, Bechtel N, Raselli C et al (2007) Fatigue in multiple sclerosis: relation to depression, physical impairment, personality and action control. Mult Scler 13(9):1161-1167

75. Petajan JH, Gappmaier E, White AT et al (1996) Impact of aerobic training on fitness and quality of life in multiple sclerosis. Ann Neurol 39(4):432-441

76. Piper AJ (2008) Sleep and quality of life in neuromuscular disease. In: Verster JC, Pandi-Perumal SRSDL (eds) Sleep and quality of life in clinical medicine. Humana Press, Totowa, pp 209-220

77. Ranjith G (2005) Epidemiology of chronic fatigue syndrome. Occup Med (Lond) 55(1):13-19

78. Rekand T, Gramstad A, Vedeler CA (2009) Fatigue, pain and muscle weakness are frequent after Guillain-Barre syndrome and poliomyelitis. J Neurol 256(3):349-354

79. Rietberg MB, Brooks D, Uitdehaag BM et al (2005) Exercise therapy for multiple sclerosis. Cochrane Database Syst Rev (1):CD003980

80. Samitz G, Bachl N (1991) Physical training programs and their effects on aerobic capacity and coronary risk profile in sedentary individuals. Design of a long-term exercise training program. J Sports Med Phys Fitness 31(2):283-293 
81. Schillings ML, Kalkman JS, Janssen HM et al (2007) Experienced and physiological fatigue in neuromuscular disorders. Clin Neurophysiol 118(2):292-300

82. Schillings ML, Stegeman DF, Zwarts MJ (2005) Determining central activation failure and peripheral fatigue in the course of sustained maximal voluntary contractions: a model-based approach. J Appl Physiol 98(6):2292-2297

83. Servaes P, van der WS, Prins J et al (2001) Fatigue in diseasefree cancer patients compared with fatigue in patients with chronic fatigue syndrome. Support Care Cancer 9(1):11-17

84. Sheean GL, Murray NM, Rothwell JC et al (1997) An electrophysiological study of the mechanism of fatigue in multiple sclerosis. Brain 120(Pt 2):299-315

85. Sivak ED, Ahmad M, Hanson MR et al (1987) Respiratory insufficiency in adult-onset acid maltase deficiency. South Med J 80(2):205-208

86. Skomro RP, Kryger MH (1999) Clinical presentations of obstructive sleep apnea syndrome. Prog Cardiovasc Dis 41(5):331-340

87. St Clair GA, Lambert ML, Noakes TD (2001) Neural control of force output during maximal and submaximal exercise. Sports Med 31(9):637-650

88. Staub F, Bogousslavsky J (2001) Fatigue after stroke: a major but neglected issue. Cerebrovasc Dis 12(2):75-81

89. Swanink CM, Vercoulen JH, Bleijenberg G et al (1995) Chronic fatigue syndrome: a clinical and laboratory study with a well matched control group. J Intern Med 237(5):499-506

90. Talbot K, Stradling J, Crosby J et al (2003) Reduction in excess daytime sleepiness by modafinil in patients with myotonic dystrophy. Neuromuscul Disord 13(5):357-364

91. Tam SL, Archibald V, Tyreman N et al (2002) Effect of exercise on stability of chronically enlarged motor units. Muscle Nerve 25(3):359-369

92. Valko PO, Bassetti CL, Bloch KE et al (2008) Validation of the fatigue severity scale in a Swiss cohort. Sleep 31(11):1601-1607

93. van Capelle CI, Winkel LPF, Hagemans MLC et al (2008) Eight years experience with enzyme replacement therapy in two children and one adult with Pompe disease. Neuromuscul Disord 18(6):447-452

94. van den Hout HMP, Hop W, van Diggelen OP et al (2003) The natural course of infantile Pompe's disease: 20 original cases compared with 133 cases from the literature. Pediatrics 112(2):332-340

95. van der Kooi EL, Kalkman JS, Lindeman E et al (2007) Effects of training and albuterol on pain and fatigue in facioscapulohumeral muscular dystrophy. J Neurol 254(7):931-940

96. Van der Ploeg AT (2005) Monitoring of pulmonary function in Pompe disease: a muscle disease with new therapeutic perspectives. Eur Respir J 26(6):984-985

97. van der Werf SP, Jongen PJ, Nijeholt GJ et al (1998) Fatigue in multiple sclerosis: interrelations between fatigue complaints, cerebral MRI abnormalities and neurological disability. J Neurol Sci 160(2):164-170

98. Van Doorn PA, Ruts L (2004) Treatment of chronic inflammatory demyelinating polyneuropathy. Curr Opin Neurol 17(5):607-613

99. Van Doorn PA, Ruts L, Jacobs BC (2008) Clinical features, pathogenesis, and treatment of Guillain-Barre syndrome. Lancet Neurol 7(10):939-950

100. Vercoulen JH, Hommes OR, Swanink CM et al (1996) The measurement of fatigue in patients with multiple sclerosis. A multidimensional comparison with patients with chronic fatigue syndrome and healthy subjects. Arch Neurol 53(7):642649

101. Vercoulen JH, Swanink CM, Fennis JF et al (1994) Dimensional assessment of chronic fatigue syndrome. J Psychosom Res 38(5):383-392

102. Vollestad NK (1997) Measurement of human muscle fatigue. J Neurosci Methods 74(2):219-227

103. Wintzen AR, Lammers GJ, van Dijk JG (2007) Does modafinil enhance activity of patients with myotonic dystrophy? A double-blind placebo-controlled crossover study. J Neurol 254(1):26-28

104. Wokke JH (2007) Fatigue is part of the burden of neuromuscular diseases. J Neurol 254(7):948-949

105. Wokke JH, Ausems MG, van den Boogaard MJ et al (1995) Genotype-phenotype correlation in adult-onset acid maltase deficiency. Ann Neurol 38(3):450-454

106. Yoshii F, Takahashi H, Kumazawa R et al (2006) Parkinson's disease and fatigue. J Neurol 253(Suppl 7):VII48-VII53

107. Zwarts MJ, Bleijenberg G, van Engelen BG (2008) Clinical neurophysiology of fatigue. Clin Neurophysiol 119(1):2-10 